CLINICAL STUDY

\title{
Genetic and environmental determinants of circulating resistin level in a community-based sample
}

\author{
I Pantsulaia ${ }^{1,2}$, G Livshits $^{1}, \mathrm{~S}$ Trofimov ${ }^{1}$ and E Kobyliansky ${ }^{1, *}$ \\ ${ }^{1}$ Department of Anatomy and Anthropology, Sackler Faculty of Medicine, Tel Aviv University, Ramat Aviv, 69978, Tel Aviv, Israel and ${ }^{2}$ Department of \\ Biomedicine, Institute of Medical Biotechnology, Tbilisi, Georgia
}

(Correspondence should be addressed to E Kobyliansky; Email: anatom14@post.tau.ac.il)

*The Lillian and Marcel Pollak chair in Biological Anthropology, Sackler Faculty of Medicine

\begin{abstract}
Objective: Resistin is a hormone secreted by adipose tissue, monocytes, bone marrow, and other tissues. It was also proclaimed as an important link between obesity and diabetes. The main objective of this study was to elucidate the contribution of a number of endogenous factors, such as sex, age, obesity characteristics, and genetic effects to the production of resistin in apparently healthy individuals. We also tested the possible relationships between circulating levels of resistin and other adipokines (leptin, interleukin-6 (IL-6), and tumor necrosis factor- $\alpha$ (TNF- $\alpha$ )).

Measurements: The plasma levels of studied adipokines were determined by enzyme-linked immunoassay in pedigree-based sample $(n=616)$, and subjected to model-based quantitative genetic analysis.

Results: Resistin levels were significantly higher in women than in men $(3.60 \pm 2.53$ vs $3.15 \pm$ $2.48 \mathrm{ng} / \mathrm{ml}, P<0.001$ ), and varied independently of age in either sex. Statistical-genetic analysis revealed significant familial correlations $(P<0.01)$ for resistin. Adjusted for covariates, $66.38 \pm$ $10.28 \%$ of the resistin variation was attributable to putative genetic factors. A relatively small portion of the resistin variation $(11.54 \pm 5.77 \%)$ was attributable to sharing a common household environment. The remaining variation, $22.12 \pm 17.69 \%$ was due to random environmental (i.e., unmeasured non-additive genetic) effects. The results of our analysis showed modest significant correlation of resistin with TNF- $\alpha$ and IL-6, and only in some groups; thus, while resistin was correlated with TNF- $\alpha$ in men, the correlation with IL- 6 was significant only in the post-menopausal women group.

Conclusions: Our observations indicate that resistin is strongly influenced by genetic factors. The high heritability estimates for resistin concentrations clearly suggest the continuing need for further molecular genetic investigations.
\end{abstract}

European Journal of Endocrinology 156 129-135

\section{Introduction}

It is now recognized that adipose tissue secretes a variety of biologically active proteins termed 'adipokines,' such as resistin, leptin, adiponectin, tumor necrosis factor- $\alpha$ (TNF- $\alpha$ ), and interleukin-6 (IL-6), which may contribute to the development of obesity-mediated adverse effects on glucose and lipid metabolism (1-4). It is also likely that some of these adipokines are specific fat-derived hormones that affect human energy homeostasis and insulin action and may be involved in hemopoiesis and immunity.

Resistin is a signal molecule generated by white adipose tissue, mammary glands, heart, brain, lungs, and the gastrointestinal tract (5). It is probably linked with inflammation, energy homeostasis, and insulin resistance (3-6), yet the available data on it are controversial. Serum levels of resistin were found to be elevated in rodent models of obesity and diabetes, suggesting a disregulation of resistin in these disease states $(5,6)$. Circulating resistin levels were also elevated in ob/ob ${ }^{-}, \mathrm{db} / \mathrm{db}^{-}$, and diet-induced obese mice (7), but another study reported decreased resistin mRNA levels in white adipose tissue of various mouse models of obesity (8). Resistin protein is detectable in human serum too, and its circulating level was found to be elevated proportionally to the degree of obesity $(9,10)$. Moreover, Zhang et al. (11) observed correlation of circulating levels of resistin with the calculated area under the glucose curve, as well as percentage of body fat and the insulinsensitivity index. Furthermore, Yannakoulia et al. (10) reported that circulating resistin protein correlated positively with body mass and negatively with waistto-hip ratio in healthy individuals. Moreover, it has been found that the release of resistin from human explants of omentum was $250 \%$ greater than that from s.c. adipose tissue (12). Collectively, these studies suggest that 
resistin is expressed in adipose tissue but not necessarily by adipocytes $(10,12)$, and may play a role in the regulation of insulin sensitivity.

That said, resistin was initially encountered in allergic pulmonary inflammation in mice and Stumvoll et al. (13) suggested that resistin might be involved in sub-clinical inflammatory processes. Subsequent studies $(14,15)$ have shown that the expression of proteins involved in the inflammatory response is enhanced in obesity states, implying that the observed metabolic abnormalities may be associated with chronic inflammation.

The above-cited clinical and experimental studies thus suggest possible multi-level involvement/association of adiposity hormones and cytokines (e.g. IL-6, TNF- $\alpha$ ) and the development of obesity, insulin resistance, and inflammation. Yet, several important questions pertaining to the variation factors of resistin circulatory levels and their relationship to pro-inflammatory cytokines in healthy humans remain unanswered. In particular, there is only one brief report, which evaluated the heritability of serum resistin and its genetic correlation with insulin resistance-related features in non-diabetic unrelated Caucasians (16).

Hence, the major aims of the present study were: (1) to evaluate in what extent putative genetic factors may contribute to variation of resistin plasma concentration in an ethnically homogeneous sample of pedigrees; and (2) to examine potential relationships between circulating levels of resistin and other relevant cytokines, particularly, leptin, TNF- $\alpha$, and IL-6. It is by now well established that sex hormones play a key role in the incipience of obesity and there are grounds for believing that they also exert a significant influence on adipokine levels (17). Hence, we deemed it worthwhile to ascertain whether they influence significantly resistin variation also.

\section{Materials and methods}

\section{Sample}

The present study was carried out on Chuvashian individuals sampled in several small villages along the Volga River (Chuvashia and Bashkortostan Autonomies, Russian Federation). Data were collected from 616 apparently healthy individuals aged 18-80 years and belonging to 145 nuclear and more complex threegeneration families. The majority of pedigrees included 2 parents with 1 to 3 offspring each, e.g. the number of families with 3 or 4 members was 119, while families with 5 or more members numbered 26 . In total, the sample available for genetic analysis consisted of 145 marital couples, 589 parent-offspring pairs and 182 sibships.

The Chuvasha ethnic group was formed during the last quarter of the first millennium in the forested or hilly portions of the Volga riverside; their ancestors were likely Bulgars from the Volga and Kama riversides, who intermarried with local Finno-Ugric tribes (18). The chosen population is characterized by demographically stable structure with traditional relations between family members. El'chinova and Ginter (19) define this population as ethnically isolated with minimal genetic flow. The gathered information entailed gender, age, basic socio-economic parameters, and standard anthropometric measurements. Subjects, who participated in the study, had no chronic or acute infection, hematological or other metabolic diseases. They were not receiving prescription medication or non-steroidal antiinflammatory drugs on a regular basis or consuming vitamin, mineral, or other dietary supplements. Reading and interpreting the medical record data, as well as interviewing study participants regarding their health status, were carried out by an experienced physician.

The data on Chuvashian pedigrees were collected during the joint expedition undertaken by the Department of Anatomy and Anthropology, Sackler Faculty of Medicine, Tel Aviv University, Israel, and the Anthropological Institute and Museum of Moscow State University, Russia. Dr Batsevich from Moscow State University supervised all the field studies. The studied families were picked randomly, through direct contact of the research group staff with practically all households in the small villages where the present project was carried out. Every subject, who agreed to participate in the study, signed an informed consent document. The project was approved by the Tel Aviv University ethics committee.

\section{Blood sampling and biochemical assays}

Venous blood samples obtained by venipuncture from overnight-fasting individuals underwent centrifugation within $1 \mathrm{~h}$ after collection. Plasma samples were immediately separated and stored in aliquots at $-80{ }^{\circ} \mathrm{C}$ for subsequent analysis. Resistin levels were determined by a sandwich enzyme immunoassay (ELISA) technique using the set of specific antibodies and standards from R\&D systems (Minneapolis, MN, USA). This system uses a solid-phase MAB and an enzyme-linked polyclonal antibody against recombinant resistin. The absorption was measured at $405 \mathrm{~nm}$ with a correction of $650 \mathrm{~nm}$ using a microplate reader (Elx808, Bio-Tek Instruments, Winooski, Vermont, USA). The detection limit of the resistin assay was $<31.25 \mathrm{pg} / \mathrm{ml}$, while the inter- and intra-assay coefficients of variation were $<5$ and $8.7 \%$ respectively.

To access plasma levels of the other cytokines, namely leptin, IL-6, and TNF- $\alpha$, we also used ELISA kits (Quantikine, R\&D Systems, Minneapolis, Minnesota, USA) with detection limits of $<8 \mathrm{pg} / \mathrm{ml}$ for leptin, $0.32 \mathrm{pg} / \mathrm{ml}$ for TNF- $\alpha$, and $0.094 \mathrm{pg} / \mathrm{ml}$ for IL- 6 . The intra- and inter-assay coefficients of variation were $<4$ and $6 \%$ respectively. Further technical information on all the assays used in this study was recently reported elsewhere $(20,21)$. 


\section{Obesity characteristics (OB)}

OB measures taken from each individual included: (i) body mass index (BMI) $\left(\mathrm{kg} / \mathrm{m}^{2}\right)$; (ii) eight skinfolds (SK) from the body trunk and extremities (chest, abdomen, subscapular, hip etc.); (iii) nine circumferences (CR), including the chest and various levels of the upper and lower extremities (waist, hip, upper arm, etc.). All the data were obtained with standard anthropometric techniques and reported in details by us earlier (22). We need to point out that, while anthropometric variables are not direct measures of adiposity but rather indirect estimates of body fat, they are widely used in this type of studies.

\section{Statistical and genetic analysis of the data}

Preliminary analysis. Statistical analyses were performed using the STATISTICA 6.0 for PC (Statsoft, Inc., Tulsa, Oklahoma, USA). All data were given as mean \pm S.D. Since the distributions of the studied molecules were markedly skewed, the data on biochemical markers were log-transformed to correct for non-normality, prior to further analysis. Review of the distribution properties characterizing the studied molecules showed some of the values to be outliers, that is, inconsistent with the distribution of the bulk of the data. To ensure that some rare observations were not ultimately excluded, we used \pm 4 s.D. (and not 3 s.D.) as the range criterion for outliers; all the relevant blood samples were now re-assayed before any final exclusion from further analysis. Differences in means of the plasma adipokines between male and female groups were determined by the MannWhitney U-test. In the female sample, all correlations with potential covariates were first examined separately for pre- and post-menopausal women, and then for the total group. However, since the correlations in the preand post-menopausal women were not significantly different (all $P>0.05$ ), the results of our genetic analysis are presented for the entire female sample combined.

Principal component analysis (PCA). PCA was undertaken on each of the two categories of $\mathrm{OB}$ traits namely skinfolds and circumferences, thereby avoiding the pitfall of multiple comparisons and redundancy of information. PCA entails a mathematical procedure that transforms a large number of correlated variables into a smaller number of uncorrelated variables called principal components (PCs). The original data, regardless of sex and age, were used in the PCA. Since the bilateral measurements of skinfolds were especially strongly correlated $(r=0.91)$, the corresponding values for each individual were averaged. The eigen value 1 criterion was utilized to retain the components. The rationale behind this criterion is straightforward to wit: any PC that yields an eigen value
$>1.0$ is accounting for a greater amount of variance than was constrained by one original variable. Thus, new composite variables, $\mathrm{PC}-\mathrm{SK}$, and $\mathrm{PC}-\mathrm{CR}$, reflecting variation of skinfolds and circumferences respectively, were used in our subsequent analyses.

Quantitative genetic analysis. In order to assess the heritability of the studied adipokines spontaneous production, genetic analysis was carried out in two stages: first, the parent-offspring correlations were computed using the statistical package Mendelian Analysis Package (MAN-5) (23), and, next the heritability $\left(h^{2}\right)$ of each trait was calculated, using standard variance components analysis based on a general quantitative genetic theory (24). Accordingly, the total variation of the phenotype $\left(V_{\mathrm{PH}}\right)$ could be divided into genetic $\left(V_{\mathrm{GN}}\right)$ and environmental $\left(V_{\mathrm{EN}}\right)$ components. Heritability is a proportion of phenotypic variation attributable to putative genetic effects, i.e., $h^{2}=V_{\mathrm{GN}} /$ $V_{\mathrm{PH}}$. The implemented method also enables to distinguish between different independent environmental components that contribute to the variation of the trait. In our general model, the environmental influences comprised variance components attributable to marital environment $\left(V_{\mathrm{SP}}\right)$, common household environment $\left(V_{\mathrm{HS}}\right)$, and specific environment shared by sibling $\left(V_{\mathrm{SB}}\right)$. The remaining unexplained residual variation was defined as $V_{\mathrm{RS}}$. Variance decomposition analysis was performed using the FISHER statistical package (25). The pertinent procedural details have been provided in numerous previous publications (e.g. (26)). The $P$ values for the heritability estimates are obtained by likelihood ratio tests, where the likelihood of a model is estimated and compared with the likelihood of a model in which the heritability is constrained to zero. Heritability of resistin levels was calculated with simultaneous adjustment for covariates such as sex, age, body weight, and obesity characteristics.

\section{Results}

\section{Descriptive statistics}

Basic descriptive statistics of the assayed individuals are summarized in Table 1, where the data are presented before log-transformation, in the original units and according to sex, and for women according to menopausal status. The span of variation of all the variables in the present study was within the range of normal variation for each sex. The plasma resistin concentration in the entire sample ranged from 1 to $17.68 \mathrm{ng} / \mathrm{ml}$. The mean levels of resistin displayed a marked sex dependency, with women having higher concentrations than men $(3.60 \pm 2.53$ vs $3.15 \pm 2.48 \mathrm{ng} / \mathrm{ml}$ respectively; 
Table 1 Descriptive statistics (mean \pm s.D.(range)) for studied variables in the Chuvashian sample (data presented according to sex and women's menopausal status).

\begin{tabular}{lcccc}
\hline & & \multicolumn{2}{c}{ Females } & \\
\cline { 3 - 4 } Variable & \multicolumn{1}{c}{ Males $(n=315)$} & \multicolumn{2}{c}{ Premenopausal $(n=171)$} & Postmenopausal $(n=130)^{\text {Mann-Whitney U-test }^{*}}$ \\
\hline Age (years) & $44.52+16.67(18.0-80.0)$ & $33.29+9.47(18.0-50.0)$ & $62.01+6.79(51.0-80.0)$ & \\
BMl & $23.13+3.32(16.28-32.76)$ & $22.83+3.67(15.77-31.74)$ & $26.23+3.83(16.57-32.94)$ & $P<0.001$ \\
PC-CR & $0.038+0.864(-2.06+2.75)$ & $-0.30+0.92(-2.27$ to 2.51$)$ & $0.31+0.989(-1.68$ to 2.62$)$ & $P<0.001$ \\
PC-SK & $-0.61+0.54(-1.51$ to 1.10$)$ & $0.41+0.83(-1.11$ to 3.13$)$ & $0.80+0.88(-0.85$ to 2.83$)$ & $P<0.001$ \\
IL-6 $(\mathrm{pg} / \mathrm{ml})$ & $1.94+1.30(0.33-6.00)$ & $1.67+1.30(0.36-6.46)$ & $2.00+1.20(0.63-6.23)$ & $P<0.001$ \\
TNF- $\alpha(\mathrm{pg} / \mathrm{ml})$ & $3.87+4.24(0.38-28.19)$ & $3.43+2.97(0.42-15.7)$ & $3.97+5.14(0.53-29.66)$ & \\
Leptin $(\mathrm{ng} / \mathrm{ml})$ & $1.89+1.60(0.10-9.01)$ & $8.42+6.78(0.84-35.87)$ & $10.03+9.05(0.78-55.57)$ & $P<0.001$ \\
Resistin $(\mathrm{ng} / \mathrm{ml})$ & $3.15+2.48(1.00-17.68)$ & $3.82+2.69(1.00-15.97)$ & $3.33+2.29(1.00-12.43)$ & $P<0.001$ \\
\hline
\end{tabular}

BMI, body mass index; PC, principal component; CR, circumferences; SK, skinfolds; IL, interleukin; TNF, tumor necrosis factor.

${ }^{\star} U$-test for comparison of males and total sample of females.

Mann-Whitney $U$-test, $P<0.001)$. The differences between pre- and post-menopausal women were nonsignificant statistically.

After the adjustment of anthropometrical traits for age, resistin levels showed consistently significant correlations with BMI, PC-CR, and PC-SK in the sample of premenopausal women (Table 2). In post-menopausal women, we found no correlation between resistin and OB measures. In men, significant correlation was detected only with PC-CR. However, the other associations with resistin were non-significant. Results of correlation analyses between plasma levels of resistin, leptin, and proinflammatory cytokines (IL-6, TNF- $\alpha$ ) indicated that only two correlations reached statistically significant level: TNF- $\alpha$ in men $(P=0.028)$ and IL-6 in postmenopausal women $(P=0.015)$. No other significant correlation was detectable in these analyses (Table 2).

\section{Familial correlations and genetic effects}

To evaluate possible familial aggregation in the variation of each of the studied biochemical variables, we computed the relevant parent-offspring correlations (Table 3). As seen from the table, familial effects were statistically significant $(P<0.001)$ for all these variables, with parent-offspring correlations ranging from 0.127 for IL- 6 to 0.385 for resistin. These findings clearly suggest the involvement of familial factors in variance of the studied cytokines.
At this stage of the study, a model-fitting analysis was applied to ascertain the extent of the genetic and environmental influences on resistin variation. Table 4 supplies the parameter estimates with their corresponding asymptotic standard errors. The table shows contribution of the potential covariates (age, height, and weight) and variance components. Likelihood ratio test clearly established that additive genetic effect on the resistin variance was highly significant $(P<0.001)$, and accounted for $66.38 \pm 10.28 \%$ of the total phenotypic variance. Common environment factors shared by spouses and sibs were constrained to zero without losing the likelihood of the model. However, common household environment influenced significantly the resistin variation $(11.54 \pm 5.77 \%)$. Genetic effects for the other studied variables ranged between $h^{2}=0.24$ and $h^{2}=0.80$, and are reported elsewhere in more detail (20). Since resistin levels showed significant correlations with IL-6 in post-menopausal women and TNF in men only, in the relatively small sized samples (Table 2), we were unable to conduct bivariate analyses.

\section{Discussion}

In the present study, we attempted to quantify the contribution of putative genetic and common environmental factors to the variance of resistin plasma levels, and to assess the dependence of this variance on age,

Table 2 Correlations between log-transformed resistin levels, obesity characteristics, and cytokines.

\begin{tabular}{lcrr}
\hline & & \multicolumn{2}{c}{ Women } \\
\cline { 3 - 4 } Variable & Men & Premenopausal & Postmenopausal \\
\hline BMl adjusted for age & $0.1234 ; P=0.083$ & $0.2217 ; P=0.018$ & $-0.1061 ; P=0.352$ \\
PC-CR adjusted for age & $0.2120 ; P=0.004$ & $0.2543 ; P=0.007$ & $-0.1479 ; P=0.212$ \\
PC-SK adjusted for age & $0.1302 ; P=0.079$ & $0.2250 ; P=0.021$ & $-0.0763 ; P=0.518$ \\
TNF- $\alpha$ & $0.1548 ; P=0.028$ & $0.0854 ; P=0.354$ & $0.0114 ; P=0.919$ \\
IL-6 & $0.0690 ; P=0.331$ & $0.0612 ; P=0.509$ & $0.2697 ; P=0.015$ \\
Leptin & $0.090 ; P=0.131$ & $0.129 ; P=0.107$ & $-0.117 ; P=0.226$ \\
\hline
\end{tabular}

BMI, body mass index; PC, principal component; CR, circumferences; SK, skinfolds; TNF, tumor necrosis factor; IL, interleukin. 
Table 3 Familial resemblance of circulating levels of cytokines in apparently healthy Chuvashian pedigrees.

\begin{tabular}{lrrrr}
\hline & Resistin & Leptin & TNF- $\alpha$ & IL-6 \\
\hline Parent/offspring correlation & 0.385 & 0.212 & 0.352 & 0.127 \\
$P$ value & $<0.001$ & $<0.001$ & $<0.001$ & 0.017 \\
\hline
\end{tabular}

TNF, tumor necrosis factor; IL, interleukin.

sex, and aforementioned cytokines (TNF $\alpha$, IL-6). Resistin expression is regulated by numerous hormones and cytokines related to the metabolism of insulin and glucose $(5,6,27)$. Despite a huge volume of recent research work, the functions and mode of actions of resistin are still not clear. Moreover, several publications studies have raised questions about the role of resistin in humans, casting doubt whether human resistin is indeed expressed in adipose tissue or represents an inflammatory marker (11, 28-30). Furthermore, there are virtually no published data on the relative contribution of genetic factors to the observed variation in circulating levels of resistin. Consequently, it is clearly important to determine circulating resistin concentrations in humans and examine their connection with systemic metabolic effects in healthy people.

The results of our study are in good conformity with the standards for healthy individuals listed in the

Table 4 Variance decomposition analysis of log-transformed resistin variation in an apparently healthy population (Chuvashian pedigrees).

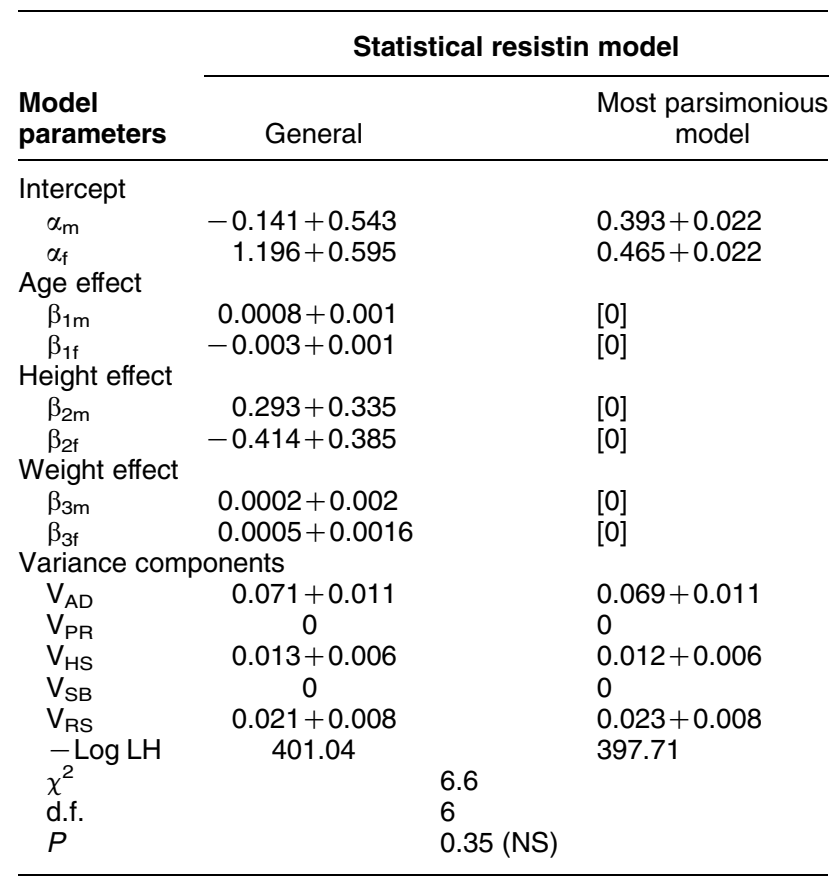

[0], parameter was fixed at the indicated value; $\alpha_{m}, \alpha_{f}$ is sex-specific intercept ( $m$, male; $f$, female); $\beta_{1}, \beta_{2}, \beta_{3}$, are sex-specific regression coefficients for age, height, and weight (respectively). Log LH, log likelihood; d.f., degrees of freedom; NS, not significant $P>0.05 ; V_{\mathrm{AD}}$, additive genetic; $V_{\mathrm{PR}}$, common parent environment; $V_{\mathrm{HS}}$, common household; $V_{\mathrm{SB}}$, common sibling; $V_{\mathrm{RS}}$. residual. instruction manual accompanying the ELISA kits we used in the present investigation. Moreover, our readings fall within the range of variation for presumably healthy subjects reported by other investigators using the same assay $(30,31)$. Just as with leptin $(10)$, so also with resistin, we confirmed that females have higher plasma levels of this protein than males $(10,32)$. However, in our sample, we failed to find any significant correlation between the circulating levels of estradiol and resistin in the female moiety (data not shown). It remains to be elucidated whether, similar to leptin, the sexual dimorphism of body fat distribution or differences in sex steroids are responsible for the observed differences in resistin levels.

Tested for possible connections between resistin variation and anthropometric characteristics of adiposity or leptin, TNF- $\alpha$ and IL- 6 has yielded only few significant correlations. In our sample, resistin variation was not dependent on BMI or skinfolds in males and post-menopausal females, which was in agreement with the study of Giannopoulou et al. (31) but in discordance with other published data (32). The literature regarding resistin levels in humans is contradictory, containing, on the one hand, reports of resistin levels being positively correlated with BMI and increase in obesity and on the other - reports that negate such correlations (32-35). Thus, Vozarova De Courten et al. (32) showed that high serum levels of resistin are related to and enhance adiposity in Pima Indians. While other investigators $(33,34)$ demonstrated that serum resistin levels did not correlate with BMI in obese individuals, even though the correlation appeared in the combined group (obese and lean subjects). The conflicting data on this issue may possibly stem from differences in protocol and experimental conditions, but to our mind and more importantly, they may reflect failure to adjust for potential confounding factors, such as gender, sex hormones, food intake, etc.

Since human resistin is likely to be involved in inflammatory processes $(14,15)$, one would expect the levels of resistin and cytokines to be correlated and indeed, Lehrke et al. (28) found a strong correlation in diabetic patients between plasma levels of resistin and STNFR2. A similar correlation between resistin and soluble receptor of TNF (sTNFR2) $(r=0.31, P<0.001)$ was found in a cohort of 879 non-diabetic individuals in the same study. Hence, in the present study, we tried to ascertain whether the resistin in normalcy correlates with other circulating cytokines. The results of our analysis revealed no reliable correlation of resistin with TNF- $\alpha$ and IL-6. We observed moderately significant correlation with TNF- $\alpha$ in male's cohort and with IL- 6 in the post-menopausal women group (Table 2). Regrettably, our corresponding sample sizes were insufficient for further bivariate analysis. The present study addressed the importance of genetic and environmental influences on the variation of resistin plasma levels in a sample of pedigrees randomly collected from apparently 
healthy European population. The statistical genetic analysis of our study evinced that the major component of the resistin variance (approximately 66\%) was attributable to familial influences, most likely due to putative genetic factors. Our results are in line with the findings by Menzhagi et al. (36), who reported that after adjusting for age and gender, serum resistin was highly heritable with almost $70 \%$ of its variability explained by genetic factors. Menzhagi et al. (36) examined the effect of three polymorphisms also ( $\mathrm{g}-20 \mathrm{C} \rightarrow \mathrm{G}$ (rs1862513), IVS2 $+181 G \rightarrow A(r s 3745367)$, and GAT(n)), but found that only IVS $2+181 \mathrm{G} \rightarrow$ A was associated with circulating ressistin explaining some $1.5 \%$ of its variation. Similar results were obtained under a recessive models, whereas none of the polymorphisms had a significant impact using a dominant model. Nevertheless, the effect of this single-nucleotide polymorphisms (SNP) is rather small, accounting for only $1.5 \%$ of resistin variance (36). Several SNPs have also been identified in the resistin gene but only a few come with minor allele frequencies over $5 \%$, and thus could provide useful information in association studies $(37,38)$. Other studies investigated associations of the given gene polymorphism with obesity and type 2 diabetes yielding scant positive results $(37,38)$. They studied four previously identified noncoding single-nucleotide polymorphisms $(-420 \mathrm{C}>\mathrm{G}$ from the promoter region, $+156 \mathrm{C}>\mathrm{T}$ and $+298 \mathrm{G}>\mathrm{A}$ from intron 2 , and $+1084 \mathrm{G}>\mathrm{A}$ from the $3^{\prime} \mathrm{UTR}$ ) and revealed that the SNPs were not significantly associated with type 2 diabetes. However, SNPs $-420 \mathrm{C}>\mathrm{G}$, $+156 \mathrm{C}>\mathrm{T}$, and $+298 \mathrm{G}>\mathrm{A}$ and the common haplotype for these three markers were associated with increased values of weight-related traits and diastolic blood pressure in cases, lower weight in elderly control subjects, and lower insulin sensitivity and greater acute insulin response in spouses (37). Finally, note Osawa et al. (39) study, who found that in healthy volunteers monocyte resistin mRNA was positively correlated with its simultaneous serum levels and was higher in G/G genotype. However, the study involved only 23 healthy individuals and therefore clearly requires confirmation on independent new samples.

In conclusion, the obtained high heritability estimates for resistin concentrations clearly suggest the ongoing need for further molecular genetic investigations that hopefully will yield successful identification of the specific genes involved.

\section{Acknowledgements}

This study was supported jointly by the Israel National Science Foundation (Grant no. 1042-04) and by a Postdoctoral Fellowship Grant (P.I.) kindly provided by UNESCO and Israel (the Ministry of Education, the Council for Higher Education's Planning and Budgeting Committee, the Ministry of Foreign Affairs and Israel National Commission for UNESCO).

\section{References}

1 Hofbauer KG. Molecular pathways to obesity. International Journal of Obesity 200226 (Suppl 2) S18-S27.

2 Paquot N \& Tappy L. Adipocytokines: link between obesity, type 2 diabetes and atherosclerosis. Review of Medicine $2005 \mathbf{6 0}$ 369-373.

3 Savege DB, Sewter CP, Klenk ES, Segal DG, Vidal-Puig A, Considine RV \& O'Rahilly S. Resistin/Fizz3 expression in relation to obesity and peroxisome proliferator activated receptor-gamma action in humans. Diabetes 200150 2199-2202.

4 Vendrell J, Broch M, Vilarrasa N, Molina A, Gomez JM, Gutierrez C, Simon I, Soler J \& Richart C. Resistin, adiponectin, ghrelin, leptin, and proinflammatory cytokines: relationships in obesity. Obesity Research 200412 962-971.

5 Adeghate E. An update on the biology and physiology of resistin. Cellular and Molecular Life Sciences 200461 2485-2496.

6 Steppan CM, Bailey ST, Bhat S, Brown EJ, Banerjee RR, Wright CM, Patel HR, Ahima RS \& Lazar MA. The hormone resistin links obesity to diabetes. Nature 2001409 307-312.

7 Way JM, Gorgun CZ, Tong Q, Uysal KT, Brown KK, Harrington WW, Oliver WR Jr, Wilson TM, Kliewer SA \& Hotamisligil GS. Adipose tissue resistin expression is severely suppressed in obesity and stimulated by peroxisome proliferator-activated receptor gamma agonists. Journal of Biological Chemistry 2001276 25651-25653.

8 Milan G, Granzotto M, Scarda A, Calcagno A, Pagano C, Federspil G \& Vettor R. Regional adipose tissue differences of resistin and adiponectin expression in genetically obese rats: effect of weight loss. Obesity Research 200210 1095-1103.

9 Degawa-Yamauchi MBJE, Juliar BE, Watson W, Kerr K, Jones RM, Zhu Q \& Considine RV. Serum resistin (FIZZ3) protein is increased in obese humans. Journal of Clinical Endocrinology and Metabolism 200388 5452-5455.

10 Yannakoulia M, Yiannakouris N, Bluher S, Matalas AL, KlimisZacas D \& Mantzoros CS. Body fat mass and macronutrient intake in relation to circulating soluble leptin receptor, free leptin index, adiponectin, and resistin concentration in healthy humans. Journal of Clinical Endocrinology and Metabolism 200388 1730-1736.

11 Zhang JL, Qin YW, Zheng X, Qiu JL \& Zou DJ. Serum resistin level in essential hypertension patients with different glucose tolerance. Diabetes 200320 828-831.

12 Fain JN, Cheema PS, Bahouth SW \& Lloyd Hiler M. Resistin release by human adipose tissue explants in primary culture. Biochemical and Biophysical Research Communications 2003300 674-678.

13 Stumvoll M \& Haring H. Resistin and adiponectin - of mice and men. Obesity Research 200211 1197-1199.

14 Park HS, Park JY \& Yu R. Relationship of obesity and visceral adiposity with serum concentrations of CRP, TNF-alpha and IL-6. Diabetes Research and Clinical Practice 200569 29-35.

15 Maachi M, Pieroni L, Bruckert E, Jardel C, Fellahi S, Hainque B, Capeau J \& Bastard JP. Systemic low-grade inflammation is related to both circulating and adipose tissue TNF-alpha, leptin and IL-6 levels in obese women. International Journal of Obesity 200428 993-997.

16 Menzaghi1 C, Coco A, Salvemini L, Thompson S, De Cosmo S, Doria A \& Trischitta V. Heritability of serum resistin and its genetic correlation with insulin resistance-related features in non-diabetic Caucasians. Journal of Clinical Endocrinology and Metabolism 2006 $912792-2795$.

17 Gui Y, Silha JV \& Murphy LJ. Sexual dimorphism and regulation of resistin, adiponectin, and leptin expression in the mouse. Obesity Research 20049 1481-1491.

18 Tischkov VA. People of Russia. Encyclopedia Moscow: Great Russian Encyclopedia Publishing House [in Russian], 1994.

19 El'chinova GI \& Ginter EK. Characterization of the marriage structure and migration in Chuvasha population. Genetika 2001 37 536-539.

20 Pantsulaia I, Trofimov S, Kobyliansky E \& Livshits G. Genetic and environmental influences on IL-6 and TNF-alpha plasma levels in apparently healthy general population. Cytokine $200219138-146$. 
21 Livshits G, Pantsulaia I, Trofimov S \& Kobyliansky E. Genetic variation of circulating leptin is involved in genetic variation of hand bone size and geometry. Osteoporosis International 200314 476-483.

22 Livshits G, Yakovenko K, Ginsburg E \& Kobyliansky E. Genetics of human body size and shape: pleiotropic and independent genetic determinants of adiposity. Annals of Human Biology 199825 221-236.

23 Malkin I, Ginsburg E. Program package for Mendelian analysis of pedigree data (MAN, Version 5). Department of Anatomy and Anthropology, Sackler Faculty of Medicine, Tel Aviv University. Technical Report. 2004.

24 Falconer DS \& Mackay TF. Introduction to Quantitative Genetics. 4 London: Longman, 1996 pp 145-284.

25 Lange K, Weeks D \& Boehnke M. Program for pedigree analysis: MENDEL, FISHER and dGENE. Genetic Epidemiology $1988 \mathbf{5}$ 471-472.

26 Livshits G, Pantsulaia I, Trofimov S \& Kobyliansky E. Genetic influences on the circulating cytokines involved in osteoclastogenesis. Journal of Medical Genetics 200441 e76.

27 Kaser S, Kaser A, Sandhofer A, Ebenbichler CF, Tilg H \& Patsch JR. Resistin messenger-RNA expression is increased by proinflammatory cytokines in vitro. Biochemical and Biophysical Research Communications $2003309286-290$.

28 Lehrke M, Reilly MP, Millington SC, Iqbal N, Rader DJ \& Lazar MA. An inflammatory cascade leading to hyperresistinemia in humans. PLoS Medicine 20042 e45.

29 McTernan PG, McTernan CL, Chetty R, Jenner K, Fisher FM, Laver MN, Crocker J, Barnett AH \& Kumar S. Increased resistin gene and protein expression in human abdominal adipose tissue. Journal of Clinical Endocrinology and Metabolism $2002 \mathbf{8 7}$ 2407-2410.

30 Wolfe BE, Jimerson DC, Orlova C \& Mantzoros CS. Effect of dieting on plasma leptin, soluble leptin receptor, adiponectin and resistin levels in healthy volunteers. Clinical Endocrinology (Oxf) 200461 332-338.

31 Giannopoulou I, Fernhall B, Carhart R, Weinstock RS, Baynard T, Figueroa A \& Kanaley JA. Effects of diet and/or exercise on the adipocytokine and inflammatory cytokine levels of postmenopausal women with type 2 diabetes. Metabolism $2005 \mathbf{5 4}$ 866-875.
32 Vozarova de Courten B, Degawa-Yamauchi M, Considine RV \& Tataranni PA. High serum resistin is associated with an increase in adiposity but not a worsening of insulin resistance in Pima Indians. Diabetes 200453 1279-1284.

33 Azuma K, Katsukawa F, Oguchi S, Murata M, Yamazaki H, Shimada A \& Saruta T. Correlation between serum resistin level and adiposity in obese individuals. Obesity Research $2003 \mathbf{8}$ 997-1001.

34 Silha JV, Krsek M, Skrha JV, Sucharda P, Nyomba BL \& Murphy LJ. Plasma resistin, adiponectin and leptin levels in lean and obese subjects: correlations with insulin resistance. European Journal of Endocrinology $2003149331-335$.

35 Lee JH, Chan JL, Yiannakouris N, Kontogianni M, Estrada E, Seip R, Orlova C \& Mantzoros CS. Circulating resistin levels are not associated with obesity or insulin resistance in humans and are not regulated by fasting or leptin administration: cross-sectional and interventional studies in normal, insulin-resistant, and diabetic subjects. Journal of Clinical Endocrinology and Metabolism $2003884848-4856$.

36 Menzaghi C, Coco A, Salvemini L, Thompson R, De Cosmo S, Doria A \& Trischitta V. Heritability of serum resistin and its genetic correlation with insulin resistance-related features in nondiabetic caucasians. Journal of Clinical Endocrinology and Metabolism 2006 $912792-2795$.

37 Conneely KN, Silander K, Scott LJ, Mohlke KL, Lazaridis KN, Valle TT, Tuomilehto J, Bergman RN, Watanabe RM, Buchanan TA, Collins FS \& Boehnke M. Variation in the resistin gene is associated with obesity and insulin-related phenotypes in Finnish subjects. Diabetologia $2004 \mathbf{4 7} 1782-1788$.

38 Ma X, Warram JH, Trischitta V \& Doria A. Genetic variants at the resistin locus and risk of type 2 diabetes in Caucasians. Journal of Clinical Endocrinology and Metabolism 200287 4407-4410.

39 Osawa H, Onuma H, Ochi M, Murakami A, Yamauchi J, Takasuka T, Tanabe F, Shimizu I, Kato K, Nishida W, Yamada K, Tabara Y, Yasukawa M, Fujii Y, Ohashi J, Miki T \& Resistin H. Resistin SNP-420 determines its monocyte mRNA and serum levels inducing type 2 diabetes. Biochemical and Biophysical Research Communications 2005335 596-602.

Received 11 July 2006

Accepted 9 October 2006 